\title{
Strongly Coupled Artificial Bulk HTS Grain Boundaries With High Critical Current Densities
}

\author{
N. Hari Babu, T. D. Withnell, K. Iida, and D. A. Cardwell
}

\begin{abstract}
A multi-seeding process has been developed to fabricate single $\mathrm{Y}-\mathrm{Ba}-\mathrm{Cu}-\mathrm{O}$ (YBCO) grains containing strong artificial grain boundaries. Multi-seeding of heterogeneous YBCO grains with controlled orientation was achieved using large $\mathrm{Sm}-\mathrm{Ba}-\mathrm{Cu}-\mathrm{O}$ (SmBCO) single crystal seeds of rod-like geometry with slots of various widths (up to $13 \mathrm{~mm}$ ) cut into their bottom surface (i.e. parallel to the c-axis of the seed) to produce a bridge-like structure. Several YBCO grains with artificial grain boundaries were fabricated from these seed crystals and used to investigate the effect of varying the distance between the individual grain nucleation sites and the grain orientation (in-plane and out of plane) on the nature of grain boundaries. The measured local magnetic critical current density $\left(J_{c}\right)$ and the magnitude of the trapped field across these artificial grain boundaries indicate that seed alignment is a key parameter in achieving strongly-coupled grain boundaries in multi-seeded grains.
\end{abstract}

Index Terms-Artificial grain boundaries, high temperature superconductors, multi-seeding, Y-Ba-Cu-O.

\section{INTRODUCTION}

$\mathbf{M}$ ELT processed single grains of $\mathrm{Y}-\mathrm{Ba}-\mathrm{Cu}-\mathrm{O}$ (YBCO) have been shown to trap a stable magnetic field as high as $17 \mathrm{~T}$ at $29 \mathrm{~K}$ and $1.5 \mathrm{~T}$ at $77 \mathrm{~K}$, which underlines the potential of bulk large grain superconductors for engineering applications [1]-[3]. Large, single grains are commonly processed using a top seeded melt growth (TSMG) technique, in which a single seed crystal is used to promote heterogeneous grain nucleation in the required orientation (typically with the $c$-axis parallel to the sample thickness). The TSMG technique has been developed considerably over the past decade and a large number of single grains of square or short cylinder geometry can now be batch-processed routinely for use in applications such as superconducting magnetic bearings [2]. For more complex devices, however, it is necessary to develop materials with good field trapping properties in specific geometries such as cylindrical cavities and long bars that can be fabricated economically if a wide range of industrial applications of these materials is to be realized. Such conformal geometries invariably require individual melt processed single crystals (grains) to be joined, or

Manuscript received August 28, 2006. This work was supported by the EPSRC.

The authors are with the IRC in Superconductivity, Engineering Department, University of Cambridge, Cambridge, CB3 0HE, U. K. (e-mail: nhb24@cam.ac.uk; tdw25@cam.ac.uk; ki227@cam.ac.uk; dc135@cam.ac. uk).

Color versions of one or more of the figures in this paper are available online at http://ieeexplore.ieee.org.

Digital Object Identifier 10.1109/TASC.2007.899063 bulk samples to be melt processed by a multi-seeding technique [4]-[9]. In either case, a good superconducting joint or clean grain boundary is required for high current or field trapping applications. Large samples have been fabricated successfully using a multi-seeding technique by Schatzle et al. [4]. This group reported a maximum trapped field of $250 \mathrm{mT}$ at $77 \mathrm{~K}$ in joined grains of size $25 \mathrm{~mm} \times 20 \mathrm{~mm}$, but with a strong suppression of the trapped field in the region between the grains. In contrast to the orientation mis-match in thin-film bi-crystals grown under vacuum, the $c$-axes of individual bulk grains either side of the grain boundary (GB) are usually well oriented to each other. Even so, the bulk GB is observed frequently to form a weak-link to current flow due primarily to the presence of residual second phases along the grain interface. Jee et al. [5] reported that the magnitude of the trapped field at the position of the join of multi-seeded YBCO grains varies inversely with the separation of the seeds and exhibits a minimum at this point when the separation between seed crystals is large. Individual grain nuclei merge together to form a single nucleation site if the inter-seed distance is small or when individual seeds are arranged in contact. In this case, the sample grows as a single grain and no residual liquid phase present in the grain microstructure. Jooss et al. [6] used a magneto-optical (MO) imaging technique to show that the quality of grain boundaries fabricated with an inter-seed distance of less than $4 \mathrm{~mm}$ is improved significantly compared to samples grown with a higher inter-seed distance. This suggests that weak-link free multi-grain superconductors can be fabricated for relatively small inter-seed distances. Haindl et al. [9] have investigated the trapped field and magneto-scan profiles produced by a magnetized superconducting ring fabricated using 10 randomly $a / b$-plane oriented, $c$-axis aligned seed crystals. Their results suggest that the misorientation angle between adjacent domains associated with the seed arrangement leads to poor grain-grain connectivity. A trapped field of $0.4 \mathrm{~T}$ at $77 \mathrm{~K}$ has been observed in multi-grains of size $\sim 5 \mathrm{~mm} \times 5 \mathrm{~mm}$. This remarkably high field, considering the small grain size, suggests that grain boundaries are coupled electrically. A multi-seeding technique has been used successfully to produce weak-link free GBs in (RE)-Ba-Cu-O from two or more various $\mathrm{RE}-\mathrm{Ba}-\mathrm{Cu}-\mathrm{O}$ layers with different peritectic temperatures [10]. In our previous study [7], the effects of seed orientation on the formation of grain boundaries and the homogeneity of multi-seeded YBCO samples was observed to correlate with the angle between the $a b$ growth sectors, with even small angles of intersection producing significant minima in the trapped field profile in the region of the GB. In this study, we report the fabrication 
of a range of large, multi-grain bulk superconductors using a multi-seeding technique and investigate the microstructure, field trapping ability and critical current density nature of the grain boundaries.

\section{EXPERIMENTAL}

$\mathrm{YBa}_{2} \mathrm{Cu}_{3} \mathrm{O}_{7}$ (Y-123) and $\mathrm{Y}_{2} \mathrm{BaCuO}_{5}$ (Y-211) precursor powders $(99.9 \%$ phase purity) with a starting composition of 70 wt $\% \mathrm{Y}-123+30$ wt $\% \mathrm{Y}-211+0.15$ wt $\%$ Pt were mixed thoroughly and pressed uniaxially into pellets of diameter $25 \mathrm{~mm}$ and thickness $15 \mathrm{~mm}$. TSMG was used to fabricate YBCO multi-grains with artificial grain boundaries using a temperature profile similar to that of described in [11]. Seed crystals with the required shape and size were cut from large, single $\mathrm{Sm}-\mathrm{Ba}-\mathrm{Cu}-\mathrm{O}$ grains fabricated using a practical melt textured processing method described in [12]. Multi-seeding experiments were carried out by varying (i) the angle $(\theta)$ between two seeded grains by using two, individual seed crystals, (ii) the distance between two nuclei whilst maintaining $\theta=0$ and (iii) the number of seed nuclei. A few natural grain boundaries (illustrated schematically in Fig. 1(a)) were fabricated by varying $\theta$ between 0 and $25^{\circ}$ with the use of two individual seeds $(\sim 2 \mathrm{~mm} \times 2 \mathrm{~mm} \times 0.5 \mathrm{~mm})$ separated by a distance of $\sim 2 \mathrm{~mm}$. In each case the angle between the two $a b$ plane growth sectors was estimated using optical microscopy from the difference in angle between individual growth facet lines of the multi-seeded samples. Several large, single seeds of slab-like geometry prepared with slots of various widths cut into their bottom surface (i.e. parallel to the c-axis of the seed) were fabricated to produce a single bridge (i.e. with a leg at each end to form two nucleation points). These bridge-shaped seed crystals simulate a multi-seeding process in which the orientation between two adjacent grains can be controlled precisely to be $\theta=0$. The geometry of the seed crystal cut from the Sm-Ba-Cu-O single grain is shown in Fig. 1(b). The distance between two seed contact points, $d$, was varied between $0.43 \mathrm{~mm}$ and $13 \mathrm{~mm}$. The top view of artificial grain boundary fabricated by TSMG is shown schematically in Fig. 1(c).

The trapped magnetic field at the sample surface was measured using a scanning Hall probe technique. This involved field cooling each sample in $500 \mathrm{mT}$, removing the field and scanning the Hall probe over the sample surface. Some samples were cut along their $a c$ plane after trapped field measurement and polished to investigate the microstructural features of the grain boundary. Small samples containing grain boundaries were cut into rectangular specimens of size $2 \mathrm{~mm} \times 1.5 \mathrm{~mm} \times 0.5 \mathrm{~mm}$ for critical current density measurements. $\mathrm{J}_{\mathrm{c}}(\mathrm{B})$ for these samples was derived from measured magnetic moment hysteresis loops.

\section{RESULTS AND DiscUSSION}

Figs. 1(d), 1(f), and 1(h) show photographs of samples fabricated by TSMG with bridge-shaped seed crystals for $d=$ $3 \mathrm{~mm}, 8.6 \mathrm{~mm}$ and $13 \mathrm{~mm}$, respectively. The cross-section and plan view of each seed crystal can be seen clearly in the figure. Figs. 1(e), 1(g), and 1(i) were obtained after removing the seed crystal from each sample. The artificial grain boundaries form as a result of impeding $a c$-planes of individual grains nucleating at different seed contact points. A sample grown from a
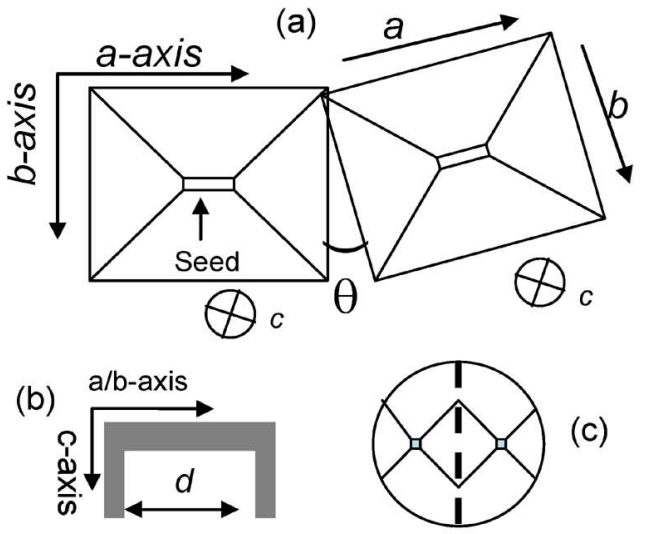

(c)
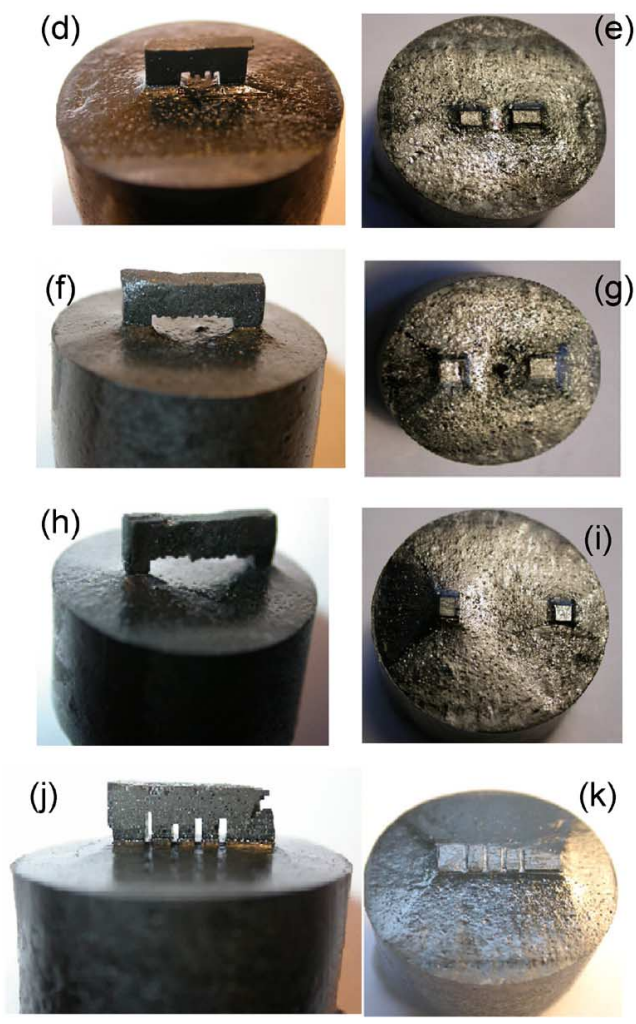

Fig. 1. (a) Schematic illustration of the top view of multi-seeded grains. (b) the shape of the seed crystal used to fabricate oriented grains and (c) the top view of an oriented grain produced with the bridge-shaped seed shown in (b). (d)-(k) Show various samples containing single and multi-grain boundaries. The photographs on the right were taken after removing seeds from as-grown samples to reveal growth facet lines. All samples are $\sim 2 \mathrm{~cm}$ in diameter.

five-leg seed (i.e. with 4 cuts made in the surface of the seed to define 5 separate points of seed contact with the surface of the precursor pellet) is shown in Fig. 1(j). The top surface of this sample following removal of seed is shown in Fig. 1(k). Two individual seeds were used in a separate TSMG process to obtain a grain boundary with a non-zero misorientation angle (i.e. $\theta \neq 0$ ). A large crack at the position of the GB was observed in most of the samples with $\theta \neq 0$, as shown in Fig. 2(a) for a sample with $\theta \sim 25^{\circ}$. In this case the crack is observed to propagate along the entire GB after the oxygenation process. A clean, crack-free $\mathrm{GB}$ without un-reacted $\mathrm{Ba}-\mathrm{Cu}-\mathrm{O}$ phase contamination was observed for samples with $0.43 \mathrm{~mm}<d<9 \mathrm{~mm}$. It is interesting to note that recognizable amounts of $\mathrm{Cu}-\mathrm{O}$ rich 

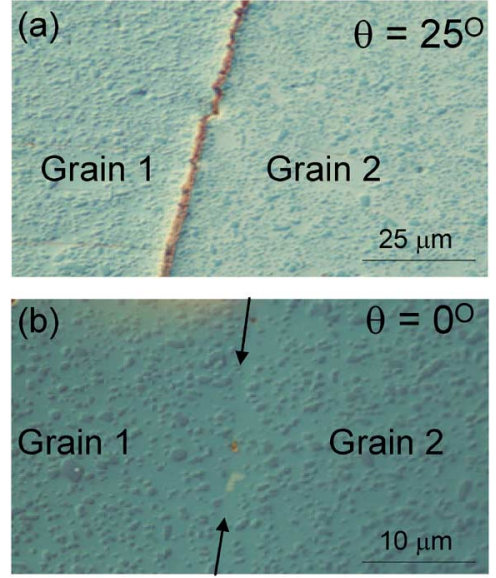

Fig. 2. Optical micrographs of grain boundaries (a) misoriented by $25^{\circ}$ within the $a b$-plane and (b) the sample shown in Fig. (1d). A clean, relatively featurefree boundary can be seen in (b).

pools and voids measuring $10-20 \mu \mathrm{m}$ were observed along the GB for higher values of $d$ (above $11 \mathrm{~mm}$ ), although the major part of the grain boundary is clean. Similar $\mathrm{Cu}-\mathrm{O}$ rich regions have been observed by Jooss et al. [6] and Jee et al. [5]. It is known that small Y-211 particles can be pushed by the Y-123 growth front and, as a result, the concentration of the Y-211 phase in the pro-peritectic melt ahead of the each growth front increases and leads to the formation of a Y-211 rich diffusion barrier. The Y-211 barrier can impede the flow of $\mathrm{Ba}$ and $\mathrm{Cu}$ ions to the growth front, which can either reduce the growth rate or stop completely growth of the Y-123 phase, depending on concentration of Y-211. It is important to note that when two growth fronts are about to impede each other during the solidification process the melt should be cooled relatively slowly to allow enough time for the $\mathrm{Ba}-\mathrm{Cu}-\mathrm{O}$ liquid to react with $\mathrm{Y}-211$ to suppress the formation of $\mathrm{Ba}-\mathrm{Cu}-\mathrm{O}$ liquid precipitates. The area fraction of Y-211 inclusions in the vicinity of grain boundary was observed to increase from $22 \%$ for $d=3 \mathrm{~mm}$ to $38 \%$ for $d=11 \mathrm{~mm}$ due primarily to particle pushing by the Y-123 growth front.

In an earlier study, the trapped field of a multi-grain sample processed with mis-oriented seeds, (e.g. for $\theta=15^{\circ}$ ), revealed the weak-link nature of the GB [7]. Fig. 3 shows the trapped field across the grain boundary for three values of $d$ when $\theta=0$. The seed crystal position and the GB are marked in the figure. Single, inverted parabolic flux profiles were observed for $d=$ $3 \mathrm{~mm}$ and $8.6 \mathrm{~mm}$, as shown in Fig. 2(b), which suggests that these GBs support large currents. The critical current density of the grain boundary $\mathrm{J}_{c, \mathrm{gb}}$, is nearly equal to that of the grain, $\mathrm{J}_{c, \mathrm{~g}}$. A minimum in trapped field is observed in the vicinity of GB when $d=13 \mathrm{~mm}$, as shown in Fig. 3(c), suggesting that $\mathrm{J}_{c, \mathrm{gb}}<\mathrm{J}_{\mathrm{c}, \mathrm{g}}$ for this GB. The magnitude of trapped field is only $10 \%$ lower than that of a sample fabricated with a single seed, suggesting that $\mathrm{J}_{c, \mathrm{gb}}$ is very high, unlike for the case of a weakly coupled GB [4], [7] (i.e. in which the trapped field along the GB is negligible). Fig. 4 shows the maximum trapped field obtained on the surface of a magnetized sample as a function of $d$. The trapped field for most of the samples containing a single artificial grain boundary is almost constant at about $0.39 \mathrm{~T}$, with

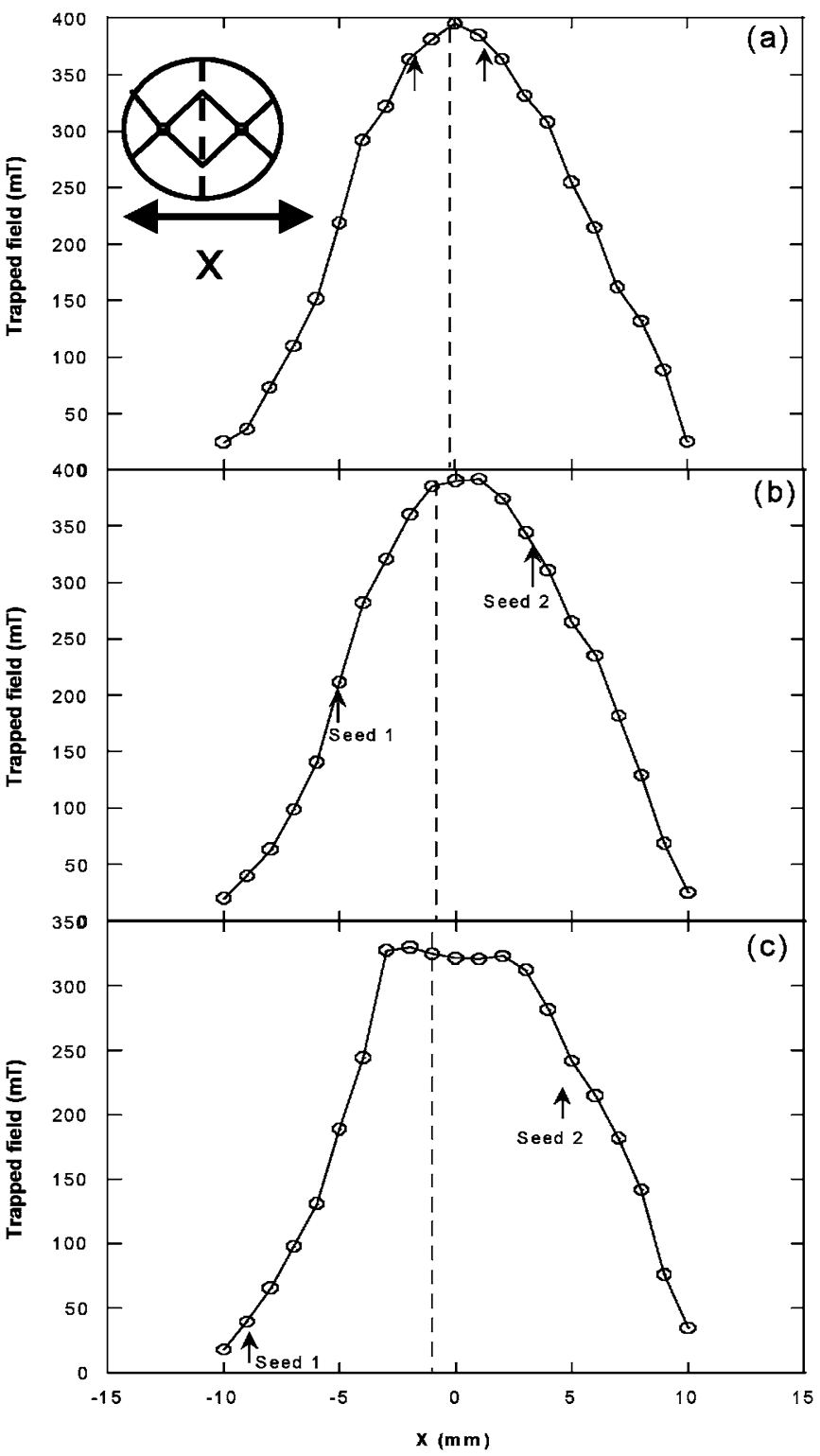

Fig. 3. Trapped magnetic profile at $77 \mathrm{~K}$ measured across a grain boundary for samples fabricated with bridge-shaped seed crystal with (a) $d=3 \mathrm{~mm}$ (b) $d=8.6 \mathrm{~mm}$ and (c) $d=13 \mathrm{~mm}$. The dashed lines and arrows indicate the position of the GB and seed contact points, respectively.

a small variation observed from sample to sample. For a sample with 4 artificial GBs, shown in Figs. 1(k) and 1(l), the trapped filed profile is again inverse parabolic in shape with a maximum trapped field of $0.41 \mathrm{~T}$ [7]. This suggests that $\mathrm{J}_{\mathrm{c}, \mathrm{gb}}$ for GB's with $\theta=0$ are nearly constant and are close to that of $\mathrm{J}_{\mathrm{c}, \mathrm{g}}$.

Magnetic moment hysteresis loops of specimens cut from the GB region of a multi-grain sample fabricated with an inter-seed distance of $11 \mathrm{~mm}$ were measured at $77 \mathrm{~K}$ to evaluate $\mathrm{J}_{c, g}$ and $\mathrm{J}_{\mathrm{c}, \mathrm{gb}}$. Fig. 5(a) shows the magnetic moment as a function of applied field for a specimen with a GB passing through its center (Sample 1), half of Sample 1 cut along its GB (Sample 2) and a specimen formed by re-joining Sample 1 with insulating varnish (Sample 3). Although the volume of Samples 1 and 3 are equal, the magnetic moment, which scales generally with the product of critical current density, $\mathrm{J}_{\mathrm{c}}$, and the length scale on which the current flows, is the largest for the sample 


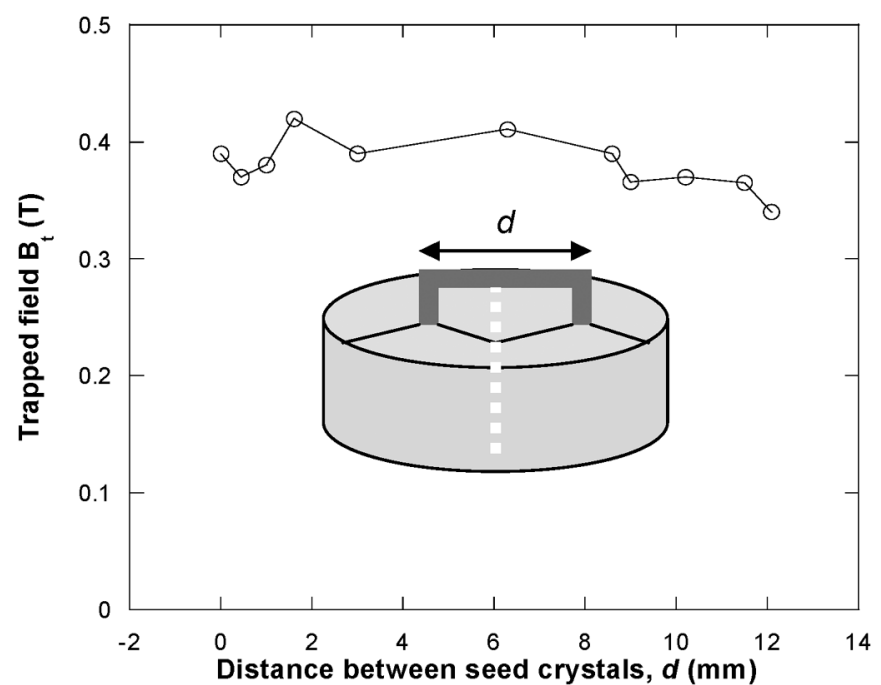

Fig. 4. Maximum trapped field as a function of seed distance.

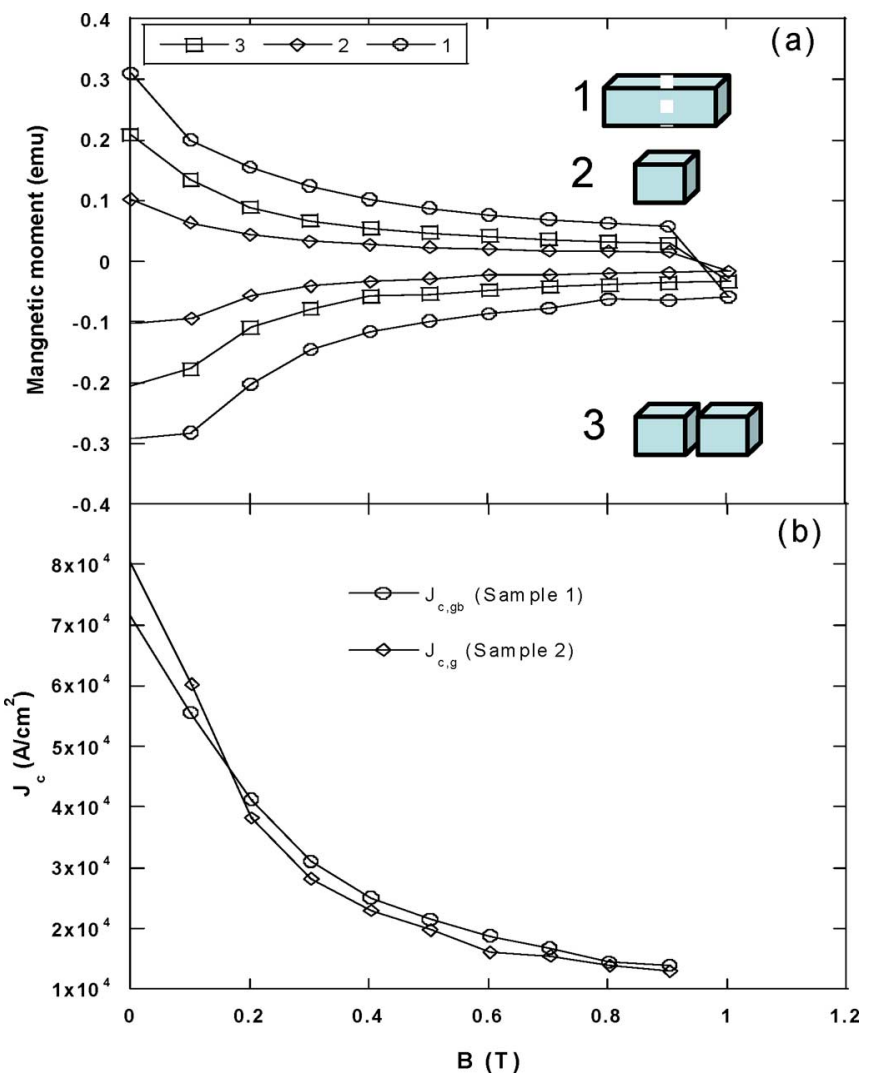

Fig. 5. (a) Magnetic moment as a function of external magnetic field at $77 \mathrm{~K}$ for samples 1, 2 and 3 (see text for details); (b) $\mathrm{J}_{\mathrm{C}}$ as a function of applied field. $\mathrm{J}_{\mathrm{c}, \mathrm{gb}}$ is nearly equal to $\mathrm{J}_{\mathrm{c}, \mathrm{g}}$.

with the artificial GB (Sample 1). This suggests that the two grains are strongly coupled. Fig. 5(b) shows $\mathrm{J}_{\mathrm{c}}(\mathrm{B})$, estimated from the measured magnetic hysteresis loops using the expression $J_{c}=20 \Delta \mathrm{m} /(a(1-a / 3 b)) ; a<b$, where $a, b$ are the dimensions of the sample measured in $\mathrm{cm}, \Delta \mathrm{m}$ is the hysteresis width in emu $/ \mathrm{cm}^{3}$ and $\mathrm{J}_{\mathrm{c}}$ in $\mathrm{A} / \mathrm{cm}^{2} . \mathrm{J}_{\mathrm{c}, \mathrm{gb}}$ is measured to be as high as $72 \mathrm{kA} / \mathrm{cm}^{2}$, which is very close to the measured $J_{c, g}$ $\left(79 \mathrm{k} / \mathrm{Acm}^{2}\right)$. A single peak in the trapped field profile, shown in Figs. 3(a) and 3(b), indicate that $\mathrm{J}_{\mathrm{c}, \mathrm{g}} \sim \mathrm{J}_{\mathrm{c}, \mathrm{gb}}$. A dual peak in the trapped field profile with a negligible trapped field along the $\mathrm{GB}$, on the other hand, suggest that $\mathrm{J}_{\mathrm{c}, \mathrm{gb}} \ll \mathrm{J}_{\mathrm{c}, \mathrm{g}}$, [4], [7].

\section{CONCLUSION}

A multi-seeding process, combined with TSMG has been used to fabricate several YBCO grains with artificial grain boundaries. Growth-related features are observed to influence the nature of artificial grain boundaries. The measured local magnetic $\mathrm{J}_{\mathrm{c}}$ and trapped field across the artificial grain boundaries indicate that seed alignment and the inter-seed distance are key parameters in achieving strongly-coupled grain boundaries in multi-seeded grains.

\section{REFERENCES}

[1] M. Tomita and M. Murakami, "High-temperature superconductor bulk magnets that can trap magnetic fields of over $17 \mathrm{~T}$ at $29 \mathrm{~K}$," Nature, vol. 421, p. 517, 2003.

[2] S. Gruss, G. Fuchs, G. Krabbes, P. Verges, G. Stöver, K.-H. Müller, J. Fink, and L. Schultz, "Superconducting bulk magnets: Very high trapped fields and cracking," Appl. Phys. Lett., vol. 79, p. 3131, 2001.

[3] D. Litzkendorf, T. Habisreuther, J. Bierlich, O. Surzhenko, M. Zeisberger, S. Kracunovska, and W. Gawalek, "Increased efficiency of batch processed melt-textured YBCO," Supercond. Sci. Technol., vol. 18, pp. S206-S208, 2005.

[4] P. Schaetzle, G. Krabbes, G. Stover, G. Fuchs, and D. Schlafer, "Multiseeded melt crystallization of YBCO bulk materials for cryogenic applications," Supercond. Sci. Technol., vol. 12, pp. 69-76, 1999.

[5] Y. A. Jee, C.-J. Kim, T.-H. Sung, and G.-W. Hong, "Top-seeded melt growth of Y-Ba-Cu-O superconductor with multiseeding," Supercond. Sci. Technol., vol. 13, p. 195, 2000.

[6] C. Jooss, B. Bringmann, M. P. Delmare, H. Walter, A. Leenders, and H. C. Freyhardt, "Current distributions of grain boundaries in differently processed melt-textured $\mathrm{YBa}_{2} \mathrm{Cu}_{3} \mathrm{O}_{7}$," Supercond. Sci. Technol., vol. 14 , p. 260, 2001.

[7] T. D. Withnell, N. H. Babu, K. Iida, Y. Shi, D. A. Cardwell, S. Haindl, F. Hengstberger, and H. W. Weber, "The effect of seed orientation and separation on the field trapping properties of multi-seeded Y-Ba-Cu-O," Physica C, vol. 445-448, p. 382, 2006.

[8] E. S. Reddy, N. Hari Babu, K. Iida, T. D. Withnell, Y. Shi, and D. A. Cardwell, "The effect of size, morphology and crystallinity of seed crystals on the nucleation and growth of $\mathrm{Y}-\mathrm{Ba}-\mathrm{Cu}-\mathrm{O}$ single grain superconductors," Supercond. Sci. Technol., vol. 18, p. 64, 2005.

[9] S. Haindl, M. Eisterer, H. W. Weber, N. H. Babu, and D. A. Cardwell, "Grain boundaries in multi-seeded melt-grown superconductors," IEEE Trans. Appl. Supercond., vol. 15, pp. 3129-3132, 2005.

[10] M. Sawamur, M. Morita, and H. Hirano, "A new method for multiseeding RE-Ba-Cu-O superconductors," Supercond. Sci Technol., vol. 17, p. S418, 2004.

[11] N. Hari Babu, M. Kambara, Y.-H. Shi, D. A. Cardwell, C. D. Tarrant, and K. R. Schneider, "Processing and microstructure of single grain, uranium doped Y-Ba-Cu-O superconductor," Supercond. Sci. Technol, vol. 15, pp. 104-110, 2002.

[12] N. Hari Babu, Y. Shi, K. Iida, and D. A. Cardwell, "A practical processing method for the fabrication of large, single grain RE-Ba-Cu-O superconductors," Nature Materials, vol. 4, pp. 476-480, 2005. 